PREPARED FOR SUBMISSION TO JHEP

\title{
Testing the bootstrap constraints in the strange sector
}

\author{
Kirill M. Semenov-Tian-Shansky, ${ }^{a, b}$ Vladimir V. Vereshagin ${ }^{b}$ \\ ${ }^{a}$ IFPA, département AGO, Université de Liège, \\ 4000 Liège, Belgium \\ ${ }^{b}$ St.-Petersburg State University, \\ St.-Petersburg, 198504, Petrodvoretz, Russia \\ E-mail: ksemenov@ulg.ac.be, vvv@av2467.spb.edu
}

\begin{abstract}
In this paper the bootstrap conditions that follow from the general postulates of effective scattering theory (EST) are checked in the strange sector. We construct the system of tree level bootstrap constraints for the renormalization prescriptions fixing the physical content of the theory. Then we perform the numerical testing of corresponding sum rules for the parameters of strange resonances. It is shown that, generally, the bootstrap constraints turn out consistent with presently known data on the strange resonance parameters. At the same time we point out few sum rules which cannot be saturated with modern data and discuss the possible reasons for such discrepancies.
\end{abstract}




\section{Contents}

1 Introduction 1

2 Preliminaries 2

$\begin{array}{lll}3 & \text { Cauchy forms in three hyperlayers } & 6\end{array}$

$\begin{array}{lll}4 & \text { The structure of bootstrap equations } & 9\end{array}$

5 Sum rules for $K N$ spectrum parameters $\quad 12$

$\begin{array}{lll}5.1 & \text { Numerical testing of bootstrap constrains } & 12\end{array}$

$\begin{array}{ll}5.2 \text { The importance of the subtraction terms } & 14\end{array}$

$\begin{array}{lll}5.3 & \text { On slowly converging sum rules } & 16\end{array}$

6 Conclusion 21

$\begin{array}{ll}\text { A Miscellaneous } & \mathbf{2 1}\end{array}$

A.1 Kaon and Nucleon field parametrization $\quad 21$

$\begin{array}{lll}\text { A.2 Asymptotic conditions } & 22\end{array}$

$\begin{array}{ll}\text { B Compact notations } & 23\end{array}$

C The explicit expressions for generating functions 24

D Kaon-Nucleon Couplings to Resonances $\quad 25$

\section{Introduction}

In [1]-[6] it has been made an attempt to develop the formalism necessary to handle the infinite-component effective theories. A special point of that formalism is that it is destined solely for the description of scattering processes. In fact we are constructing not an effective field theory but rather an effective scattering theory (EST). The key to the solution of specific problems which emerge when one considers an infinite component effective theory is provided by the requirement of existing the rigorously defined terms (each given by an infinite series) of Dyson perturbation expansion at every fixed loop order. The constructive form of this requirement results in a set of non-trivial relations for the renormalization prescriptions (RPs) that fix the physical content of the theory - so called bootstrap constrains.

In principle, once solved the system of bootstrap constraints would give the answer to the question: "How many independent RPs are needed to fix completely the physical content of an infinite component EST?"'and would show its true predictive power. Unfortunately, actually we are unable to solve this system explicitly. However, the numerical tests of sum 
rules for the parameters of resonances derived from the bootstrap system can be used to check the consistency of EST approach.

In this paper we continue the work started in [1]-[6]. As shown in [7], the data on resonances in three channels of the elastic pion-nucleon scattering process turn out to be in nice agreement with corresponding bootstrap constraints. This gives us a hope that the main principles forming the basis for extended perturbation scheme mirror correctly the regularities of hadron spectrum. Therefore, it seems natural to check if the method of [7] leads to reasonable results in the case of strange resonances that appear in elastic kaon-nucleon scattering. This process presents special interest because, first, it is relatively well studied experimentally and, second, the resonance spectra in $s$ - and $u$-channels differ from one another. The latter circumstance provides a possibility to exploit a far more rich system of bootstrap constraints as compared to that used in our analysis of pion-nucleon elastic scattering.

The main principles of constructing the extended perturbation scheme are discussed in [1]-[6]. A detailed step-by-step instruction on their application for the case of binary processes at tree level is presented in [7]. Therefore, in this paper we refer the reader to quoted above articles for the details of our approach and focus only on the specific points of the particular case under consideration.

We would like to emphasize that the main goal of this paper is the analysis of the bootstrap relations for the parameters of strange resonances and checking their consistency with well established experimental numbers. We do not aim to give a precise description of the relevant amplitudes in any kinematical domain (say, near threshold or in the resonance region).

The paper is organized similarly to [7]. In Section 2 the basic formulae needed to construct the Cauchy forms for tree-level invariant amplitudes of $K N$ elastic scattering are presented. Next, in Section 3 we construct the Cauchy forms in three mutually intersecting hyperlayers. With these expressions in hand, in Section 4 we derive the system of bootstrap constraints (sum rules) for the set of renormalization prescriptions fixing the physical content of the effective theory. Bootstrap system restricts the values of resonance mass parameters and the minimal (resultant) triple coupling constants ${ }^{1}$. The results of numerical testing of the corresponding sum rules for spectrum parameters are discussed in Section 5. We present a large set of sum rules that are well saturated by the known data. Next we consider certain sum rules which are not saturated by the parameters of presently known resonances and discuss the possible sources of discrepancies. The conclusions are given in Section 6 .

\section{Preliminaries}

Let us consider the kaon-nucleon elastic scattering process:

$$
N_{\alpha}(k, \lambda)+K_{i}(p) \rightarrow N_{\beta}\left(k^{\prime}, \lambda^{\prime}\right)+K_{j}\left(p^{\prime}\right)
$$

\footnotetext{
${ }^{1}$ We refer the reader to Ref. [6] for the detailed explanation of the terminology.
} 
a)

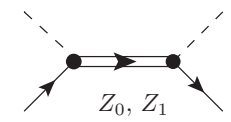

b)

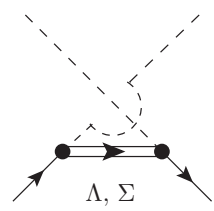

c)

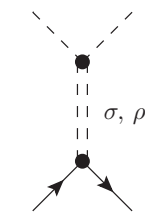

Figure 1. We need to calculate the on-shell numerators of these graphs.

Here $\lambda, \lambda^{\prime}=\{1,2\}$ stand for the nucleon spin variables and $i, j(\alpha, \beta)=\{1,2\}$ are the kaon (nucleon) isotopic indices (see Appendix A for the summary of our field conventions). The isotopic structure of the amplitude reads:

$$
M_{\alpha i}{ }^{\beta j}=i(2 \pi)^{4} \delta^{(4)}\left(p+k-p^{\prime}-k^{\prime}\right)\left\{\delta_{\alpha} \cdot{ }^{\beta} \cdot \delta_{i} \cdot{ }^{j} \cdot M^{+}\left(\lambda, \lambda^{\prime}\right)+\delta_{\alpha} \cdot{ }^{j} \cdot \delta_{i} \cdot{ }^{\beta} \cdot M^{-}\left(\lambda, \lambda^{\prime}\right)\right\} .
$$

Each one of the isotopic amplitudes $M^{ \pm}$can be presented as follows:

$$
M^{ \pm}\left(\lambda, \lambda^{\prime}, s, t, u\right)=\bar{u}\left(\lambda^{\prime}, k^{\prime}\right)\left\{A^{ \pm}(s, t, u)+\hat{Q} B^{ \pm}(s, t, u)\right\} u(\lambda, k) .
$$

Throughout the paper we adopt the Dirac "hat" notation: $\hat{p} \equiv p^{\mu} \gamma_{\mu} ; \bar{u}\left(k^{\prime}, \lambda^{\prime}\right), u(k, \lambda)$ stand for the nucleon Dirac spinors and $Q \equiv \frac{p+p^{\prime}}{2}$. The invariant amplitudes $A^{ \pm}$and $B^{ \pm}$may be considered depending on arbitrary pair of the Mandelstam variables:

$$
\begin{aligned}
& s=(k+p)^{2}, \quad t=\left(p-p^{\prime}\right)^{2}, \quad u=\left(k-p^{\prime}\right)^{2} ; \\
& s+t+u=2\left(m^{2}+\mu^{2}\right) \equiv 2 \sigma,
\end{aligned}
$$

where $m(\mu)$ stands for the nucleon (kaon) mass. We introduce the special notations for two useful combinations of mass parameters:

$$
\theta \equiv\left(M_{R}^{2}-\sigma\right), \quad \Sigma \equiv\left(M_{R}^{2}-2 \sigma\right),
$$

where $M_{R}$ is the mass parameter of a resonance $R$.

The construction of scattering amplitudes to a given loop order in the EST approach implies the use of the modified system of Feynman rules, containing only minimal (resultant) vertices and minimal propagators. Our present goal is to work out the tree level expressions for $A^{ \pm}$and $B^{ \pm}$. To make use of the technique of Cauchy forms for these amplitudes we need to specify their residues at poles corresponding to $s$-, $t$ - and $u$-channel resonance exchanges. In other words, we have to calculate the on-shell numerators in the expressions which correspond to the contributions of graphs shown on Fig. 1. For this we need to compute the products of the form $V \Pi V$, where $V$ stand for the relevant minimal triple vertices while $\Pi$ denotes the covariant spin sum (numerator of the minimal propagator) of a resonance in question.

Analogous to the case of $\pi N$ scattering [7], it is possible to construct the corresponding minimal triple vertices with the help of the listed below Hamiltonian monomials (c.f. [8]). Let us stress that this is only possible with respect to minimal vertices with three lines. The parametrization of minimal vertices with $l \geq 4$ lines could be written out explicitly only in the momentum space. 
Let us first consider the monomials that correspond to the depicted on Fig. 1b $u$-channel baryon resonance $R_{u}$ exchanges with strangeness $S=-1$ and isospin $I=0,1$ (in particular $\Lambda(1115)$ and $\Sigma(1189)$ hyperons occur in this channel). They look as follows:

$$
H\left(R_{u} \bar{K} N\right)=g_{R} P_{\rho(0,1)^{\cdot k \alpha \cdot}}^{k \cdot j} \bar{\Psi}_{\alpha} \Gamma(\mathcal{N}) R_{\cdot k \mu_{1} \ldots \mu_{l}}^{\rho \cdot} \partial^{\mu_{1} \ldots \mu_{l}} K^{j}+\text { h.c. }
$$

Here $P_{\rho(0,1)}^{\cdot k \alpha \cdot}$ are the $u$-channel isospin projectors (see (A.3)) while

$$
\Gamma(\mathcal{N})= \begin{cases}1_{4 \times 4}, & \text { for } \mathcal{N}=-1 \\ i \gamma_{5}, & \text { for } \mathcal{N}=+1\end{cases}
$$

the notation $\mathcal{N} \equiv \mathbf{P}(-1)^{l}$ is used for the normality of a resonance with spin $j=l+\frac{1}{2}$ and parity $\mathbf{P} ; g_{R}$ stands for the minimal (dimensional) coupling constant.

The Hamiltonian monomials for the vertices describing $s$-channel exchanges (with strangeness $S=1$ exotic baryon resonances) only differ from (2.4) by the isospin projectors (A.1).

We also need the Hamiltonian monomials which correspond to minimal vertices describing $t$-channel non-strange meson exchanges with isospin $I=0,1$, normality $\mathcal{N}=+1$ and $\operatorname{spin} j=l$. There are two monomials of this kind since the minimal $R N \bar{N}$ vertex contains two independent tensor structures:

$$
\begin{aligned}
& H\left(R_{t} N \bar{N}\right)=P_{\rho}^{t(0,1)}{ }_{\rho \cdot \cdot i}^{\sigma n} \cdot\left\{\frac{1}{2} f^{(1)} g_{R N \bar{N}}^{(1)} \bar{\Psi}_{\sigma} \partial^{\mu_{1} \ldots \mu_{l}} \Psi^{\rho}\right. \\
& \left.+\frac{1}{2} f^{(2)} g_{R N \bar{N}}^{(2)} j \bar{\Psi}_{\sigma} \gamma_{\mu_{j}} \partial^{\mu_{1} \ldots \mu_{l-1}} \Psi^{\rho}\right\} R_{n \cdot \mu_{1} \ldots \mu_{l}}^{\cdot i}+\text { h.c. } \\
& H\left(R_{t} K \bar{K}\right)
\end{aligned}
$$

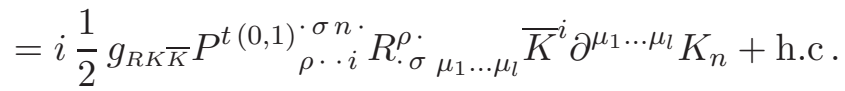

Here $P^{t(0,1)}{ }_{\rho \cdot i}^{\sigma n}$ stand for $t$-channel isospin projectors (A.2). Besides, we introduce the phase factors $f^{(1,2)}$

$$
f^{(1)}=\left\{\begin{array}{ll}
1, & \text { for } J=0,2, . . \\
i, & \text { for } J=1,3, \ldots
\end{array} ; \quad f^{(2)}= \begin{cases}i, & \text { for } J=0,2, . . \\
1, & \text { for } J=1,3, \ldots\end{cases}\right.
$$

in order to ensure hermiticty.

The numerator of minimal propagator is just a covariant spin sum. So, minimal propagators for $s$ - and $u$-channel baryon resonances read:

$$
\frac{i}{(2 \pi)^{4}} \frac{\Pi_{\xi \mu_{1} \ldots \mu_{l} ; \eta \nu_{1} \ldots \nu_{l}}(q)}{q^{2}-M_{R}^{2}+i \epsilon}
$$


where $\Pi_{\xi \mu_{1} \ldots \mu_{l} ; \eta \nu_{1} \ldots \nu_{l}}(q)$ is the covariant spin sum of the spin $j=l+\frac{1}{2}$ baryon resonance [12] $(\xi, \eta=\{1,2,3,4\}$ stand for the Dirac spinor indices). The minimal propagator for $t$-channel meson resonance differs from (2.7) only by the structure of spin sum.

The technique needed to calculate the principal parts of contributions of the graphs with spin- $j$ resonance exchanges is described in [7]; here we briefly illustrate it by way of computing the graph shown in Fig. 1 (b). Using the minimal vertices that correspond to the monomial (2.4), we obtain the following expression for the matrix element of $u$-channel exchange by the baryon resonance $R$ with the mass parameter $M_{R}$, isospin $I$ and normality $\mathcal{N}:$

$$
-P_{\alpha \cdot{ }^{u}(0,1) \cdot j \beta}^{\cdot j} g_{R}^{2} \bar{u}^{+}\left(\lambda^{\prime}, k^{\prime}\right) \Gamma \frac{(-1)^{l} \mathcal{P}_{l+\frac{1}{2}}\left(-p,-p^{\prime}\right)}{u-M_{R}^{2}} \Gamma u^{-}(\lambda, k) .
$$

Here $P^{u(0,1)^{\cdot j \beta} \cdot i}$ stands for the $u$-channel isospin projector (A.3) and $\mathcal{P}_{l+\frac{1}{2}}\left(-p,-p^{\prime}\right)$ is the contracted projector (covariant spin sum contracted with the appropriate number of $-p$ and $-p^{\prime}$ vectors).

Since all we need are the expression for the residue at $u=M_{R}^{2}$ we make use of an explicit form [12] for the contracted projector $\mathcal{P}_{l+\frac{1}{2}}\left(p^{\prime}, p\right)$ calculated between the nucleon spinors under the on-mass-shell (OMS) conditions $\left(k^{2}=k^{\prime 2}=m^{2}, p^{2}=p^{2}=\mu^{2}, u=M_{R}^{2}\right)$ :

$$
\begin{aligned}
& \left.\bar{u}^{+}\left(\lambda^{\prime}, k^{\prime}\right) \mathcal{P}_{l+\frac{1}{2}}\left(-p,-p^{\prime}\right) u^{-}(\lambda, k)\right|_{O M S} \\
& =\bar{u}^{+}\left(\lambda^{\prime}, k^{\prime}\right) \frac{l !(-1)^{l}}{(2 l+1) ! !}\left[F_{A}^{l}\left(-\mathcal{N} M_{R}, t\right)+\hat{Q} F_{B}^{l}\left(-\mathcal{N} M_{R}, t\right)\right] u^{-}(\lambda, k) .
\end{aligned}
$$

Here $F_{A, B}^{l}$ are given by the relations (B.1, B.2) (see Appendix B). With the help of (2.9) one can easily calculate the contributions of (2.8) to the principle parts of invariant amplitudes $A^{ \pm}$and $B^{ \pm}$defined in (2.3).

Computing all the elements shown on Fig. 1 one can gather the contributions of individual graphs into four invariant amplitudes $X^{ \pm}=\left\{A^{ \pm}, B^{ \pm}\right\}$and write down the final result for their principal parts. In terms of shortened notations introduced in Appendix B the formal expressions for the principal parts of the invariant amplitudes read:

$$
\begin{aligned}
\text { P.p. }\left[X^{ \pm}(s, t, u)\right] & =-\sum_{\substack{B(I=0,1) \\
S=+1}} c_{I}^{ \pm} \frac{\left.Y_{X}(\ldots, t)\right|_{s=M_{s}^{2}}}{s-M_{s}^{2}}-\sum_{\substack{B(I=0,1) \\
S=-1}} \eta_{X} b_{I}^{ \pm} \frac{\left.Y_{X}(\ldots, t)\right|_{u=M_{u}^{2}}}{u-M_{u}^{2}} \\
& -\sum_{\substack{M(I=0,1) \\
S=0}} d_{I}^{ \pm} \frac{\left.W_{X}\left(\ldots, \frac{s-u}{4 F}\right)\right|_{t=M_{t}^{2}}}{t-M_{t}^{2}} .
\end{aligned}
$$

The first two sums are taken over all possible baryon resonances with isospin $I=0,1$ and strangeness $S= \pm 1$. The third sum is taken over all possible non-strange meson resonances with isospin $I=0,1$. Let us stress that at this stage the sums in (2.10) are to be taken just as formal. The formulation of suitable convergency conditions for these formal series allows one to define rigorously the 0th (tree level) order approximation of the loop expansion for the EST amplitude in the sector of strange hadrons. 


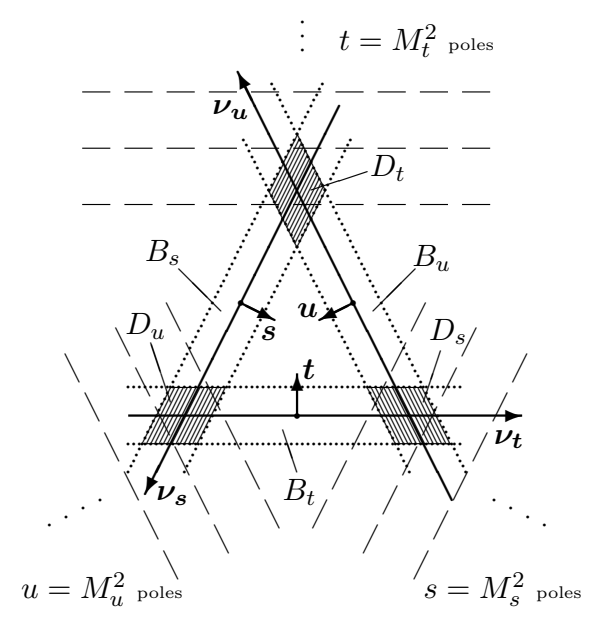

Figure 2. Mandelstam plane: three different Cauchy series converge uniformly in three different hyperlayers $B_{s}, B_{t}$ and $B_{u}$ (their sections by the Mandelstam plane are bounded by dotted lines); $\left(s, \nu_{s} \equiv u-t\right),\left(t, \nu_{t} \equiv s-u\right),\left(u, \nu_{u} \equiv t-s\right)$ are the natural coordinates in $B_{s}, B_{t}$ and $B_{u}$, respectively. The domains of the layer intersections are hatched (denoted as $D_{s}, D_{t}$, and $D_{u}$ ). Approximate positions of the poles (pole lines) in $s, t$, and $u$-channels are shown by dashed lines (the mass parameters are real).

\section{Cauchy forms in three hyperlayers}

In accordance with the summability principle [6], within our EST approach the tree level invariant amplitudes of $K N$ scattering $X^{ \pm}=\left\{A^{ \pm}, B^{ \pm}\right\}$are required to be meromorphic functions in each pair energy $\nu_{x}\left(\nu_{s} \equiv u-t, \nu_{t} \equiv s-u, \nu_{u} \equiv t-s\right)$ at arbitrary fixed value of the momentum transfer $x=\{s, t, u\}$. The uniformity principle specifies that in every hyperlayer $B_{x}:\left\{\nu_{x} \in \mathbb{C}, x \sim 0\right\}$ (see Fig. 2) containing the zero momentum transfer $(x=0)$ hyperplane, the invariant amplitudes must be polynomially bounded functions of the corresponding variable $\nu_{x}$. The bounding polynomial degree in every hyperlayer $B_{x}$ is fixed by the value of the relevant Regge intercept. The method of the Cauchy forms (that is in fact the adaptation of the conventional dispersion relation technique for the case of meromorphic functions) allows one to present the amplitude which is $N$-bounded in a hyperlayer $B_{x}$ as a uniformly converging series of pole contributions.

Let us start with constructing the Cauchy forms in the hyperlayers $B_{s}$ and $B_{u}$. The situation in these cases is trivial since in both layers all the invariant amplitudes possess decreasing asymptotic behavior (see Appendix A.2). Therefore the relevant Cauchy forms are just sums of pole contributions. This information together with the formal expressions (2.10) for principle parts of the invariant amplitudes is sufficient for constructing the well defined Cauchy forms for tree level invariant amplitudes of $K N$ scattering in these layers. The only singularities of the tree level graphs in these hyperlayers are simple poles in variables $\nu_{s}$ and $\nu_{u}$.

With the use of the compact notations introduced in Appendix B one may treat all $X^{ \pm}$on the same footing. The residues at poles which correspond to the $u$-channel baryon resonances with strangeness $S=-1$ and hypothetic exotic $s$-channel resonances with $S=$ 
+1 are given by $Y_{X}$ (B.4). The residues at poles corresponding to the $t$-channel meson resonance exchanges are given by the functions $W_{X}$ (B.6).

Thus in the layer $B_{s}$ we have:

$$
\begin{aligned}
X^{ \pm}\left(s, \nu_{s}\right) & =-\sum_{\substack{B(I=0,1) \\
S=-1}} \eta_{X} b_{I}^{ \pm} Y_{X}(\ldots,-(\Sigma+s)) \frac{2}{\nu_{s}-(s+2 \theta)} \\
& -\sum_{M(I=0,1)} d_{I}^{ \pm} W_{X}\left(\ldots, \frac{2 s+\Sigma}{4 F}\right) \frac{-2}{\nu_{s}+(s+2 \theta)},
\end{aligned}
$$

while in $B_{u}$ :

$$
\begin{aligned}
X^{ \pm}\left(u, \nu_{u}\right) & =-\sum_{\substack{B(I=0,1) \\
S=+1}} \eta_{X} b_{I}^{ \pm} Y_{X}(\ldots,-(\Sigma+u)) \frac{-2}{\nu_{u}+(u+2 \theta)} \\
& -\sum_{M(I=0,1)} d_{I}^{ \pm} W_{X}\left(\ldots, \frac{-(2 u+\Sigma)}{4 F}\right) \frac{2}{\nu_{u}-(u+2 \theta)} .
\end{aligned}
$$

In what follows we also employ the Cauchy forms (3.1) and (3.2) rewritten in terms of corresponding natural variables in the domains $D_{u}=B_{s} \cap B_{t}, D_{t}=B_{u} \cap B_{s}, D_{s}=B_{t} \cap B_{u}$.

Let us now consider the hyperlayer $B_{t}$. The Regge theory requirements listed in the Appendix A.2 provide information on the asymptotic behavior of the isotopic combinations of the invariant amplitudes $\left(2 X^{+}+X^{-}\right)$and $X^{-}$for large $\left|\nu_{t}\right|$. Thus in the layer $B_{t}$ the Cauchy forms for tree level amplitudes require introducing the correcting polynomials in $\nu_{t}$ of the degrees $N_{t}$ :

$$
\begin{array}{ll}
N_{t}\left(2 A^{+}+A^{-}\right)=1 ; & N_{t}\left(A^{-}\right)=0 ; \\
N_{t}\left(2 B^{+}+B^{-}\right)=0 ; & N_{t}\left(B^{-}\right)=-1 .
\end{array}
$$

Surely, the smooth terms - polynomials of the same degrees in $\nu_{t}$ with the coefficient functions depending on $t$ - must be taken into account.

For $A^{+}$in $B_{t}$ we put down the Cauchy form with the background term and correcting polynomials of 1 st order in $\nu_{t}$ :

$$
A^{+}\left(t, \nu_{t}\right)=\alpha_{A^{+}}^{0}(t)+\alpha_{A^{+}}^{1}(t) \nu_{t}+\widetilde{A}^{+}\left(t, \nu_{t}\right) .
$$

Here by $\widetilde{A}^{+}$we denote the principle part of the corresponding Cauchy form with the necessary correcting polynomials:

$$
\begin{aligned}
\widetilde{A}^{+}\left(t, \nu_{t}\right) & \equiv-\sum_{\substack{B(I=0,1) \\
S=+1}} Y_{A}(\ldots, t)\left(\frac{2 c_{I}^{+}}{\nu_{t}-(t+2 \theta)}+\frac{2 c_{I}^{+}}{t+2 \theta}+\frac{2 \nu_{t}\left(c_{I}^{+}+\frac{1}{2} c_{I}^{-}\right)}{(t+2 \theta)^{2}}\right) \\
& -\sum_{\substack{B(I=0,1) \\
S=-1}} Y_{A}(\ldots, t)\left(\frac{-2 b_{I}^{+}}{\nu_{t}+(t+2 \theta)}+\frac{2 b_{I}^{+}}{t+2 \theta}-\frac{2 \nu_{t}\left(b_{I}^{+}+\frac{1}{2} b_{I}^{-}\right)}{(t+2 \theta)^{2}}\right)
\end{aligned}
$$


and $\alpha_{A^{+}}^{0,1}(t)$ describe the regular at $t=0, \nu_{t}=0$ part of $A^{+}$.

The Cauchy forms for $A^{-}$in $B_{t}$ must contain the correcting polynomials and background terms of 0 th degree in $\nu_{t}$, therefore:

$$
A^{-}\left(t, \nu_{t}\right)=\alpha_{A^{-}}^{0}(t)+\widetilde{A}^{-}\left(t, \nu_{t}\right) .
$$

Here, as above, the notation

$$
\begin{aligned}
\widetilde{A}^{-}\left(t, \nu_{t}\right) & \equiv-\sum_{\substack{B(I=0,1) \\
S=+1}} c_{I}^{-} Y_{A}(\ldots, t)\left(\frac{2}{\nu_{t}-(t+2 \theta)}+\frac{2}{t+2 \theta}\right) \\
& -\sum_{\substack{B(I=0,1) \\
S=-1}} b_{I}^{-} Y_{A}(\ldots, t)\left(\frac{-2}{\nu_{t}+(t+2 \theta)}+\frac{2}{t+2 \theta}\right) .
\end{aligned}
$$

is used for the singular ("principal") part of the Cauchy form with necessary correcting polynomials.

The Cauchy form for $B^{+}\left(t, \nu_{t}\right)$ is similar to that for $A^{-}\left(t, \nu_{t}\right)$ (it also requires introducing the correcting polynomials of 0th degree in $\nu_{t}$ ):

$$
B^{+}\left(t, \nu_{t}\right)=\alpha_{B^{+}}^{0}(t)+\widetilde{B}^{+}\left(t, \nu_{t}\right) .
$$

Here

$$
\begin{aligned}
\widetilde{B}^{+}\left(t, \nu_{t}\right) & \equiv-\sum_{\substack{B(I=0,1) \\
S=+1}} Y_{B}(\ldots, t)\left(\frac{2 c_{I}^{+}}{\nu_{t}-(t+2 \theta)}+\frac{2 c_{I}^{+}+c_{I}^{-}}{t+2 \theta}\right) \\
& +\sum_{\substack{B(I=0,1) \\
S=-1}} Y_{B}(\ldots, t)\left(\frac{-2 b_{I}^{+}}{\nu_{t}+(t+2 \theta)}+\frac{2 b_{I}^{+}+b_{I}^{-}}{t+2 \theta}\right) .
\end{aligned}
$$

Finally, the Cauchy form for $B^{-}\left(t, \nu_{t}\right)$ in $B_{t}$ is just the sum of pole contributions:

$$
\begin{aligned}
B^{-}\left(t, \nu_{t}\right) & =-\sum_{\substack{B(I=0,1) \\
S=+1}} Y_{B}(\ldots, t) \frac{2 c_{I}^{-}}{\nu_{t}-(t+2 \theta)} \\
& -\sum_{\substack{B(I=0,1) \\
S=-1}} Y_{B}(\ldots, t) \frac{2 b_{I}^{-}}{\nu_{t}+(t+2 \theta)} \equiv \widetilde{B}^{-}\left(t, \nu_{t}\right) .
\end{aligned}
$$

To derive the system of bootstrap constrains we need to rewrite the Cauchy forms for $X^{ \pm}$in terms of natural variables of relevant intersection domains.

For example, in the intersection domain $D_{u} \equiv B_{t} \cap B_{s}$ the natural variables are $t$ and $s$ (both $t, s \sim 0$ ). Therefore making use of the relation $\nu_{t} \equiv s-u=t+2 s-2 \sigma$ we obtain

$$
\begin{aligned}
& \left.A^{+}\right|_{D_{u}}(t, s)=\alpha_{A^{+}}^{0}(t)+\alpha_{A^{+}}^{1}(t)(t+2 s-2 \sigma)+\widetilde{A}^{+}(t, s) ; \\
& \left.A^{-}\right|_{D_{u}}(t, s)=\alpha_{A^{-}}^{0}(t)+\widetilde{A}^{-}(t, s) ; \\
& \left.B^{+}\right|_{D_{u}}(t, s)=\alpha_{B^{+}}^{0}(t)+\widetilde{B}^{+}(t, s) ; \\
& \left.B^{-}\right|_{D_{u}}(t, s)=\widetilde{B}^{-}(t, s) .
\end{aligned}
$$


Here $\widetilde{X}^{ \pm}(X=A, B)$ stand for the singular ("principal") parts of corresponding Cauchy forms with necessary correcting polynomials rewritten in terms of natural variables of the given intersection domain. We adopt the following convention on the order of arguments of $\widetilde{X}^{ \pm}$: the natural variable which marks the hyperlayer where the initial Cauchy form was written (in the case under consideration this is $B_{t}$ ) stands at the first position.

\section{The structure of bootstrap equations}

The tree level bootstrap conditions follow from the requirement that every tree level invariant amplitude must be a meromorphic function with definite asymptotic behavior in hyperlayers $B_{s}, B_{t}$ and $B_{u}$. Hence three different Cauchy forms which present the amplitude in these layers must coincide pairwise in the relevant intersection domains $D_{s}, D_{t}$ or $D_{u}$. As pointed out in [6], the bootstrap conditions restrict the allowed values of the tree-level resultant parameters. It is these parameters which stand in the right hand sides of renormalization prescriptions fixing the physical content of effective scattering theory in the renormalization scheme without oversubtractions. Once resolved the (full) system of tree level bootstrap constraints would single out the set of essential parameters of a theory. The higher level bootstrap conditions only can further restrict this set.

Let us first construct the system of tree level bootstrap conditions for the invariant amplitude $A^{+}$. In each one of three intersection domains $D_{u}=B_{s} \cap B_{t}, D_{t}=B_{u} \cap B_{s}$ and $D_{s}=B_{t} \cap B_{u}$ the two different Cauchy series for $A^{+}$(see Section 3 ) are equally applicable. Thus employing the conventions of Section 3 and the notations introduced in (3.11) we have:

- In $D_{u}($ for $s, t \sim 0)$ :

$$
\left\{\begin{array}{l}
\left.A^{+}\right|_{D_{u}}=A^{+}(s, t) \\
\left.A^{+}\right|_{D_{u}}=\alpha_{A^{+}}^{0}(t)+\alpha_{A^{+}}^{1}(t)(t+2 s-2 \sigma)+\widetilde{A}^{+}(t, s) .
\end{array}\right.
$$

- In $D_{t}($ for $s, u \sim 0)$ :

$$
\left\{\begin{array}{l}
\left.A^{+}\right|_{D_{t}}=A^{+}(s, u) \\
\left.A^{+}\right|_{D_{t}}=A^{+}(u, s)
\end{array}\right.
$$

- In $D_{s}($ for $t, u \sim 0)$ :

$$
\left\{\begin{array}{l}
\left.A^{+}\right|_{D_{s}}=A^{+}(u, t) \\
\left.A^{+}\right|_{D_{s}}=\alpha_{A^{+}}^{0}(t)-\alpha_{A^{+}}^{1}(t)(t+2 u-2 \sigma)+\widetilde{A}^{+}(t, u) .
\end{array}\right.
$$

To ensure the possibility of analytic continuation from one hyperlayer to another, each pair of the relevant series must coincide identically in the intersection domain where both expansions are valid. Thus we obtain the following system of conditions: 
- In $D_{u}$ :

$$
\alpha_{A^{+}}^{0}(t)+\alpha_{A^{+}}^{1}(t)(t+2 s-2 \sigma)+\varphi_{A^{+}}(s, t)=0,
$$

where $\varphi_{A^{+}}(s, t) \equiv \widetilde{A}^{+}(t, s)-A^{+}(s, t)$.

- In $D_{t}$ :

$$
0=\Phi_{A^{+}}(u, s) \equiv A^{+}(s, u)-A^{+}(u, s)
$$

- In $D_{s}$ :

$$
\alpha_{A^{+}}^{0}(t)+\alpha_{A^{+}}^{1}(t)(-t-2 u+2 \sigma)-\Psi_{A^{+}}(t, u)=0,
$$

where $\Psi_{A^{+}}(t, u) \equiv A^{+}(u, t)-\widetilde{A}^{+}(t, u)$.

Here we have introduced three generating functions: $\varphi_{A^{+}}(s, t), \Phi_{A^{+}}(u, s)$ and $\Psi_{A^{+}}(t, u)$ (see Appendix C) which are the differences of sums of principle parts (with correcting polynomials, if needed) of two Cauchy forms in the corresponding hyperlayers. In the same way as in [2], [6] we exploit the fact that in (4.1)-(4.3) the dependence of generating functions (or their partial derivatives) on certain Mandelstam variables is purely fictitious. This allows one to express explicitly the unknown functions $\alpha$ in terms of spectrum parameters (in [6] such expressions were called the first kind bootstrap constraints). Besides, we derive the consistency conditions for these expressions (second kind bootstrap constraints). Throughout the text we adopt the following notations for partial derivatives in the Mandelstam variables $x=\{s, t, u\}$ :

$$
\left(\partial_{x}\right)^{k} \equiv \frac{\partial^{k}}{\partial x^{k}}
$$

First we note that, according to $(4.2), \Phi_{A^{+}}(u, s)$ is identically zero everywhere in the vicinity of the point $u=0, s=0$. Therefore the following consistency conditions hold:

$$
\left.\left(\partial_{u}\right)^{p}\left(\partial_{s}\right)^{k} \Phi_{A^{+}}(u, s)\right|_{\substack{u=0 \\ s=0}}=0, \text { for all } p, k=0,1,2, \ldots .
$$

Next, from (4.3) for all $t, u \sim 0$ we define the unknown background term:

$$
\alpha_{A^{+}}^{1}(t)=-\frac{1}{2}\left(\partial_{u}\right) \Psi_{A^{+}}(t, u)
$$

The condition which ensures the consistency of the definition (4.6) reads:

$$
\left.\left(\partial_{t}\right)^{p}\left(\partial_{u}\right)^{k+2} \Psi_{A^{+}}(t, u)\right|_{\substack{t=0 \\ u=0}}=0, \text { for all } p, k=0,1,2, \ldots
$$

At the same time, the same function $\alpha_{A^{+}}^{1}(t)$ can be defined from (4.1):

$$
\alpha_{A^{+}}^{1}(t)=-\frac{1}{2}\left(\partial_{s}\right) \varphi_{A^{+}}(s, t) .
$$


The consistency condition for this - alternative - definition reads:

$$
\left.\left(\partial_{s}\right)^{p+2}\left(\partial_{t}\right)^{k} \varphi_{A^{+}}(s, t)\right|_{\substack{s=0 \\ t=0}}=0, \text { for all } p, k=0,1,2, \ldots
$$

From the requirement that the expression (4.6) should not contradict to another - equally possible - expression (4.8) for the same function, we obtain the following system of equivalence conditions:

$$
\begin{aligned}
& \left(\partial_{t}\right)^{p}\left[\left(\partial_{u}\right) \Psi_{A^{+}}(t, u)_{u=0}\right]_{t=0}=\left(\partial_{t}\right)^{p}\left[\left(\partial_{s}\right) \varphi_{A^{+}}(s, t)_{s=0}\right]_{t=0} . \\
& \text { for all } p=0,1,2, \ldots \text { : }
\end{aligned}
$$

Finally, from (4.3) and (4.6) we define for all $t \sim 0$ the second unknown function $\alpha_{A^{+}}^{0}(t)$ :

$$
\alpha_{A^{+}}^{0}(t)=\Psi_{A^{+}}(t, 0)-\frac{1}{2}(t-2 \sigma)\left[\left(\partial_{u}\right) \Psi_{A^{+}}(t, u)\right]_{u=0} .
$$

Alternatively, this function can be derived from (4.1):

$$
\alpha_{A^{+}}^{0}(t)=\varphi_{A^{+}}(0, t)+\frac{1}{2}(t-2 \sigma)\left[\left(\partial_{s}\right) \varphi_{A^{+}}(s, t)\right]_{s=0} .
$$

The corresponding system of equivalence conditions reads:

$$
\begin{aligned}
\left(\partial_{t}\right)^{p}\left\{\frac { ( t - 2 \sigma ) } { 2 } \left[\left(\partial_{s}\right) \varphi_{A^{+}}\right.\right. & \left.\left.(s, t)\right|_{s=0}+\left.\left(\partial_{u}\right) \Psi_{A^{+}}(t, u)\right|_{u=0}\right] \\
& \left.+\left[\varphi_{A^{+}}(0, t)-\Psi_{A^{+}}(t, 0)\right]\right\}=0, \text { for all } p=0,1,2, . .
\end{aligned}
$$

Bootstrap constraints for $A^{-}$may be derived in the same way; they are more simple because $A^{-}$possesses constant asymptotics in the hyperlayer $B_{t}$. As above, we introduce three generating functions (see Appendix C): $\varphi_{A^{-}}(s, t), \Phi_{A^{-}}(u, s)$, and $\Psi_{A^{-}}(t, u)$. Then, the set of bootstrap constraints contains one condition of the first kind

$$
\alpha_{A^{-}}(t)=\Psi_{A^{-}}(t, 0)
$$

(it fixes the unknown function $\alpha_{A^{-}}(t)$ ) and four systems of the second kind constraints. Three of these latter systems, namely,

$$
\begin{aligned}
& \left.\left(\partial_{t}\right)^{p}\left(\partial_{u}\right)^{k+1} \Psi_{A^{-}}(t, u)\right|_{\substack{t=0 \\
u=0}}=0, \\
& \left.\left(\partial_{s}\right)^{p+1}\left(\partial_{t}\right)^{k} \varphi_{A^{-}}(s, t)\right|_{\substack{s=0 \\
t=0}}=0, \\
& \left.\left(\partial_{u}\right)^{p}\left(\partial_{s}\right)^{k} \Phi_{A^{-}}(u, s)\right|_{\substack{u=0 \\
s=0}} ^{u}=0
\end{aligned}
$$

$(p, k=0,1,2, \ldots)$ present the consistency conditions while the fourth one

$$
\left(\partial_{t}\right)^{p}\left[\Psi_{A^{-}}(t, 0)+\varphi_{A^{-}}(0, t)\right]_{t=0}=0 ; \text { for all } p=0,1,2, \ldots .
$$

ensures the equivalence of two possible definitions of $\alpha_{A^{-}}(t)$ (it plays the same role as (4.10) and (4.13)). 
To write down the bootstrap constraints for $B^{ \pm}$we introduce six generating functions: $\varphi_{B^{ \pm}}(s, t), \Phi_{B^{ \pm}}(u, s)$ and $\Psi_{B^{ \pm}}(t, u)$ (see Appendix C). The constraints for $B^{+}$are analogous to those for $A^{-}$. The system for $B^{-}$is even more simple. It consists of three second kind bootstrap conditions:

$$
\begin{aligned}
& \left.\left(\partial_{t}\right)^{p}\left(\partial_{u}\right)^{k} \Psi_{B^{-}}(t, u)\right|_{\substack{t=0 \\
u=0}}=0 ; \\
& \left.\left(\partial_{s}\right)^{p}\left(\partial_{t}\right)^{k} \varphi_{B^{-}}(s, t)\right|_{\substack{s=0 \\
t=0}}=0 ; \\
& \left.\left(\partial_{u}\right)^{p}\left(\partial_{s}\right)^{k} \Phi_{B^{-}}(u, s)\right|_{\substack{u=0 \\
s=0}}=0 ; \text { for all } p=0,1,2, \ldots
\end{aligned}
$$

Thus we have constructed the system of bootstrap conditions for the invariant amplitudes of $K N$ elastic scattering process. The first kind bootstrap constraints (4.6), (4.11) and (4.14) define the smooth parts of those amplitudes. The constraints of the second kind (namely, (4.5), (4.7), (4.9), (4.15) and (4.17)) provide the consistency conditions for these definitions. Finally, (4.10), (4.13) and (4.16) ensure that the definitions are not contradictive.

It should be noted that the above-obtained system of bootstrap constraints still is not complete. The degrees of bounding polynomials needed to construct the Cauchy forms for certain combinations of invariant amplitudes in the layers $B_{s}$ and $B_{u}$ are $\leq-2$. This results in appearing of additional super-convergence conditions (see [4]) which we are not going to consider here.

\section{$5 \quad$ Sum rules for $K N$ spectrum parameters}

\subsection{Numerical testing of bootstrap constrains}

Tree level bootstrap equations derived in the previous Section represent the set of limitations imposed by the requirement of mathematical correctness of extended perturbation scheme on the values of renormalization prescriptions (RPs) fixing the physical content of EST. Therefore starting from the tree level the bootstrap system results in non-trivial constraints for the values of physical parameters of the theory. Higher level bootstrap constraints obviously differ from those of tree level; they may impose additional constraints on the set of physical parameters.

In other words, within our EST approach the tree level bootstrap constraints are valid at any order of loop expansion and possess an important predictive power. The numerical testing of tree level bootstrap constraints is, therefore, rightful. Moreover, it is of great interest because it allows one to make at least preliminary conclusions about the consistency of basic postulates employed in EST approach such as the summability and uniformity principles with the present day phenomenology. Such a verification proved to be successful in the cases of $\pi K$ and $\pi N$ scattering (see [7], [2], [3]). Below we perform similar analysis of the bootstrap constraints for the parameters of kaon-nucleon resonance spectrum.

Despite the fact that these parameters are known with much less precision than those of pion-nucleon resonances, it still turns out possible to single out the set of sum rules that 
are well saturated by known experimental data. On the other hand, those sum rules which are not so well saturated with now existing data, permit us to speculate about possible scenarios that could amend the situation. Thus in our numerical tests we also aim to show that the extended perturbation scheme provides us with a tool to study the resonance spectrum.

In our numerical studies we make use of the data [14] on hadron spectrum (Table 1 of Appendix D). Several phenomenological constants were taken from early reviews [911]. The formulae connecting resonance couplings with the decay widths are presented in Appendix D).

Note that we did not include in Table 1 the results of recent analysis concerning the fine structure of the strange resonance spectrum (see, e.g. [15] and references therein). The reason is that the error bars induced by the data on well established lowest resonances $\Lambda$ and $\Sigma$ turn out to be larger than the possible total contribution of narrow resonances discussed in [15]. For this reason the latter contribution turns out invisible against that background.

Obviously, the existing information on the $K N$ resonance spectrum [14] is incomplete in the region of high mass and spin. Moreover, much is unclear with $M>1 \mathrm{GeV}$ meson resonances in $t$-channel of the elastic $K N$ reaction. Second, spin- $\frac{1}{2}$ resonances over the $u$-channel threshold are not so well established too. One also needs to keep in mind the possible existence of $s$-channel exotic resonances with strangeness $S=+1$. Therefore, our first goal is to find those sum rules which can be saturated with the reliable experimental data. The invariant amplitudes $X^{-}=\left\{A^{-}, B^{-}\right\}$receive contributions from the exchanges with uncharged hyperons in the $u$-channel. The main advantage is that both the $\Lambda(I=0)$ and $\Sigma(I=1)$ hyperon families contribute. Thus one may expect that due to mutual cancelations the saturation of sum rules for $X^{-}$can be achieved faster than in the case of invariant amplitudes $X^{+}$. For the latter (as a consequence of isospin invariance) only the $\Sigma(I=1)$ family of hyperons contribute in the $u$-channel.

As an example we have chosen the sum rules which follow from the bootstrap constraints of the second kind (4.15) for the invariant amplitude $A^{-}$.

$$
\left.\left(\partial_{u}\right)^{p}\left(\partial_{s}\right)^{k} \Phi_{A^{-}}(u, s)\right|_{\substack{u=0 \\ s=0}}=0, \text { for all } p, k=0,1, \ldots
$$

It turns out that for certain sum rules of this group the contributions from some poorly established resonances are not essential.

It is straightforward to check that for $p=0,1,2$ and $k=1,2$ the corresponding sum rules can be considered as purely baryonic ones. Indeed in the meson sector only isospin1 resonances of odd spin $J \geq 3$ (e.g., $\rho_{3}(1690)$ ), in principle, can contribute. We make a natural assumption that the heavy meson contributions are suppressed by small $\sim \frac{1}{M}$ factors. Next, one can check that in the $S=-1$ baryon sector only resonances with $J=\frac{3}{2}, \frac{5}{2}, \ldots$ contribute to these sum rules. In this way we also manage to evade the problem with poorly established spin $\frac{1}{2}$ resonances over the $\bar{K} N$ threshold. In our present analysis we are not going to take account of possible contributions from exotic resonances 
with strangeness $S=+1$. However, in what follows we show that several sum rules provide an indirect evidences in favor of existence of exotics.

To characterize the convergency of a given sum rule we introduce the partial sums $S^{+}\left(M_{R}\right)$ and $S^{-}\left(M_{R}\right)$ of positive and negative contributions, respectively. For example, for $\Phi_{A^{-}}(u, s)$ the partial sums are defined as:

$$
\begin{aligned}
& S^{+}(M)=\sum_{\substack{R_{s} R_{t} R_{u}, M_{R} \leq M}}\left(\partial_{u}\right)^{p}\left(\partial_{s}\right)^{k} \phi_{A^{-}}(u, s)_{\substack{u=0 \\
s=0}}, \\
& \text { where every item } \\
& S^{-}\left(\partial_{u}\right)^{p}\left(\partial_{s}\right)^{k} \phi_{A^{-}}(u, s)_{\substack{u=0 \\
s=0}} \geq 0 ; \\
& \text { where every item } \\
& \sum_{\substack{R_{s} R_{t} R_{u} \\
M_{R} \leq M}}\left|\left(\partial_{u}\right)^{p}\left(\partial_{s}\right)^{k} \phi_{A^{-}}(u, s)\right|_{\substack{u=0 \\
s=0}}, \\
& y^{p}\left(\partial_{s}\right)^{k} \phi_{A^{-}}(u, s)_{\substack{u=0 \\
s=0}}<0 .
\end{aligned}
$$

Here $\phi_{A^{-}}$stands for the individual resonance contribution to the generating function $\Phi_{A^{-}}(u, s)$. Clearly, if $S^{+} \approx S^{-}$the sum rule in question can be considered as well saturated. On Figures 3, 4, 5, 7 for different sum rules we represent the dependence of the corresponding partial sums $S^{+}$and $S^{-}$on the mass of heaviest $u$-channel resonance taken into account. The error bars for $S^{+}$and $S^{-}$originate mainly from the uncertainties of the resonance decay widths (and, hence, of triple coupling constants).

To make the domains of intersection of error bars of $S^{+}$and $S^{-}$better visible on our Figures (Fig. 3, 4, 5, 7) the error bars corresponding to $S^{-}$are shifted by $10 \mathrm{MeV}$ to the right from the true resonance position.

The results of saturation of several first sum rules that follow from the bootstrap constraints (5.1) are shown on Fig. 3. One can conclude that these sum rules seem to be very well saturated by known experimental data on the $S=-1$ baryon resonances with $J=\frac{3}{2}, \frac{5}{2}, \frac{7}{2}$ and masses $<2.4 \mathrm{GeV}$.

The similar sets of well saturated sum rules also follow from the bootstrap constraints for $B^{-}$invariant amplitude in the domain $D_{t}$ (with generating function $\Psi_{B^{-}}$) and for $A^{-}$ in $D_{s}$ (with generating function $\Psi_{A^{-}}$) and $D_{u}$ (with generating function $\phi_{A^{-}}$). There are also some other reasonably well saturated sum rules which we do not show here. It seems highly improbable that the nice agreement with data of the large number of sum rules stemming from bootstrap constraints in three distantly lying domains of the Mandelstam plane can be explained by accidental luck. So we conclude that the crucial assumptions of our EST approach at least do not contradict roughly to the known phenomenology of $K N$ scattering.

\subsection{The importance of the subtraction terms}

In order to stress the importance of the proper formulation of the uniformity principle (see Section 3) and the necessity to take account of the correcting polynomials in the Cauchy forms for invariant amplitudes in the hyperlayers with non-decreasing asymptotic behavior let us perform the following exercise. Consider the sum rules that follow from the bootstrap 

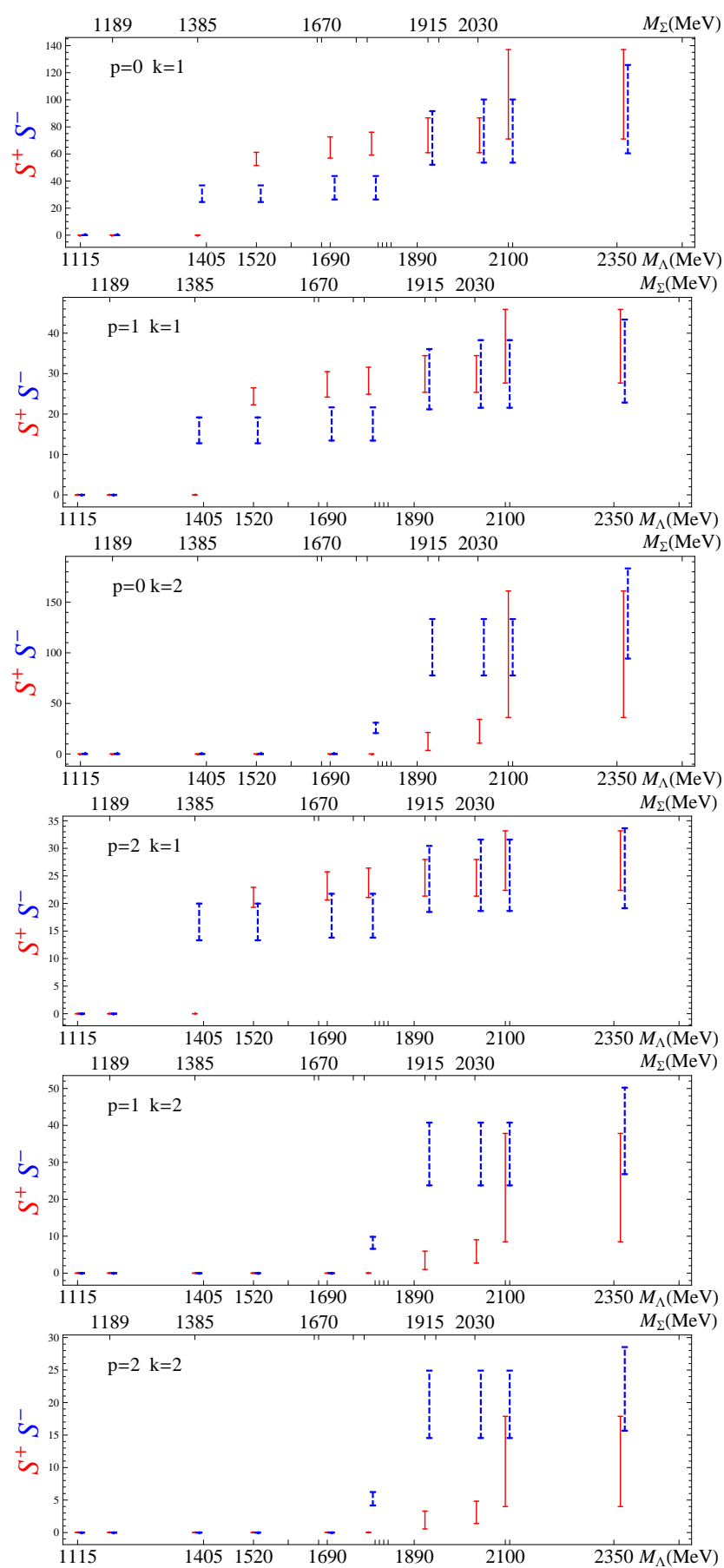

Figure 3. Numerical tests of sum rules following from bootstrap constraints (5.1) for different values of $k$ and $p . S^{+}$(solid) v.s. $S^{-}$(dashed) as functions of the heaviest $S=-1$ baryon resonance taken into account. The error bars corresponding to $S^{-}$are shifted by $10 \mathrm{MeV}$ to the right from the resonance position for better discernibility.

constraint for the invariant amplitude $A^{-}$in the domain $D_{u}$. We are going to compare the 
saturation of the sum rules obtained under the incorrect assumption that $A^{-}$has decreasing asymptotic behavior in the hyperlayer $B_{t}$ with the sum rules obtained under the correct asymptotic assumption (in fact, $A^{-}$is 0-bounded function in the hyperlayer $B_{t}$ ).

As an example we have chosen the simplest sum rule following from the requirement that the Cauchy form for $A^{-}$in the hyperlayer $B_{t}$ should coincide with that constructed in the hyperlayer $B_{s}$ at the central point $\{s=0, t=0\}$ of the domain $D_{u}$ :

$$
\left.A^{-}(t=0, s=0)\right|_{B_{t}}=\left.A^{-}(s=0, t=0)\right|_{B_{s}} .
$$

Under the incorrect assumption on the asymptotic behavior of $A^{-}$in hyperlayer $B_{t}$ the corresponding Cauchy form is just the sum over $s$ - and $u$-channel poles. On the contrary, under the proper asymptotic assumption (A.5) it involves the correcting polynomials of 0th degree in $s-u$ as well as the smooth (background) term $\alpha_{A^{-}}(t)$. The background term can be computed from the bootstrap constraint in the domain $D_{s}$ with the help of the first kind bootstrap constraint (4.14). The sum rule in question follows from the bootstrap constraint of the second kind (4.16) with $p=0$ and takes the form:

$$
\Psi_{A^{-}}(0,0)+\varphi_{A^{-}}(0,0)=0 .
$$

The results of saturation of the sum rule obtained under incorrect assumption on the asymptotic behavior of $A^{-}$in $B_{t}$ and the correct sum rule (5.3) with existing data are shown on Fig. 4. Note that this sum rule also receives contribution from the $t$-channel meson resonances with $I=1$. Because of poor knowledge of the relevant meson couplings we take account of contribution of the lightest $\rho(770)$ meson which is supposed to be dominant in the meson sector. The value of the corresponding coupling $G_{1}$ (see B.8) is taken from $[9,10]$. It is clearly visible that the sum rule following from the correct suggestion on the asymptotic behavior of $A^{-}$in the layer $B_{t}$ is much better saturated than the sum rule without subtraction term and correcting polynomials.

This example demonstrates the importance of both principles (summability and uniformity) which give rise to the system of bootstrap constraints in EST approach. Under the incorrect assumption on the asymptotic behavior of invariant amplitudes one could hardly expect to fulfil the analyticity requirements encoded in the system of bootstrap conditions.

\subsection{On slowly converging sum rules}

In Section 5.1 we presented an impressive series of well saturated sum rules for $K N$ resonance parameters following from the system of bootstrap conditions. However the situation with some other sum rules looks less optimistic. Thus we have to perform the more detailed analysis. It looks natural to discuss the possible reasons for which ceratin sum rules cannot be saturated by the presently available data.

As an example, let us consider a particular sum rule which follows from the second kind bootstrap constraints (4.5) for the invariant amplitude $A^{+}$in $D_{t}$ :

$$
\left.\left(\partial_{u}\right)^{p}\left(\partial_{s}\right)^{k} \Phi_{A^{+}}(u, s)\right|_{\substack{u=0 \\ s=0}}=0, \quad \text { for all } p, k=0,1, \ldots .
$$




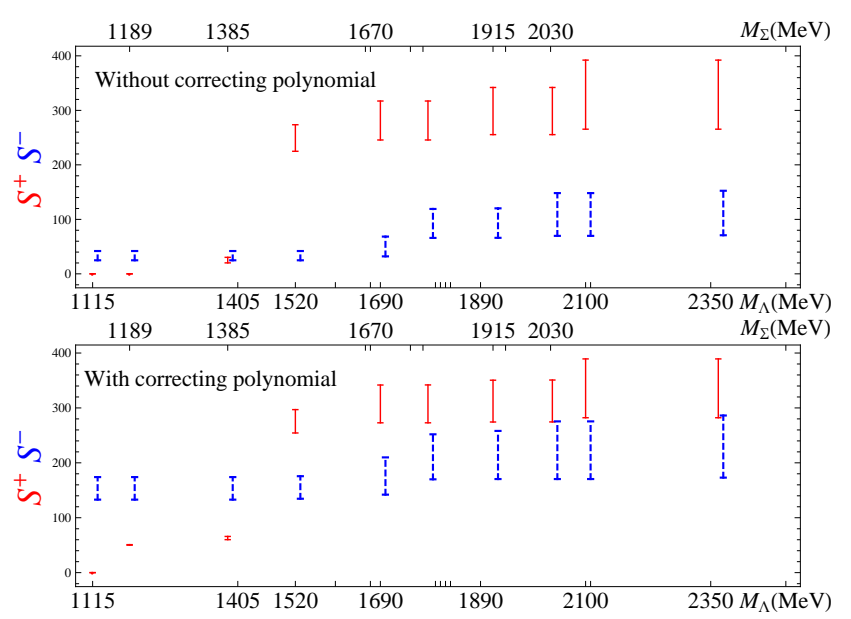

Figure 4. Numerical tests of sum rule for the invariant amplitude $A^{-}$in the domain $D_{s}$. On the upper panel we show the saturation of the sum rule (5.2) written without correcting polynomials and background term for $A^{-}$in $B_{t}$. On the lower panel we show the saturation of the sum rule (5.3) which takes account of the proper asymptotic condition for $A^{-}$in that hyperlayer.

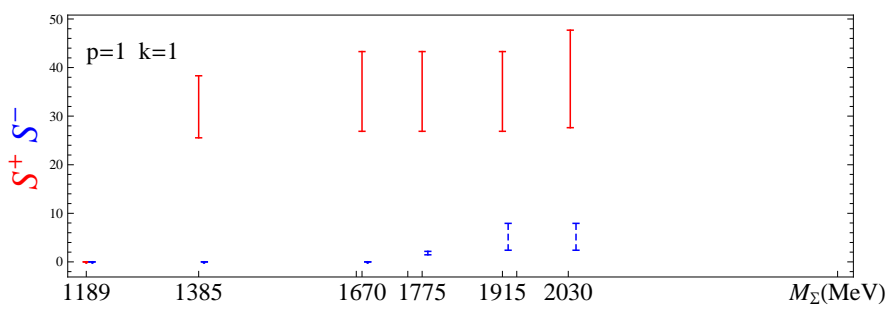

Figure 5. Numerical test of the sum rule from the system of constraints (5.4) for $k=1$ and $p=1$. Partial sums $S^{ \pm}$are shown as functions of mass parameter of the heaviest $\Sigma$ hyperon taken into account.

The results of saturation of the sum rule that follows from (5.4) at $p=k=1$ are shown on Fig. 5. $\Lambda$ hyperons do not contribute and, at first glance, nothing can compensate the huge positive contribution of $\left(I=1, J=\frac{3}{2}\right)$ resonances closest to threshold. Let us discuss the possible way to overcome this difficulty.

First of all, we would like to recall that a similar situation was encountered in the "toy bootstrap model" for the Lovelace string-like amplitude [4]. To achieve the reasonable accuracy in the course of numerical testing of certain sum rules for the resonance parameters in this model, it was sufficient to take account of the contributions of relatively small number of lowest poles. At the same time, when saturating some other sum rules it was necessary to take account of the contributions from considerable number of poles in one variable in order to compensate the "accidentally large" contribution coming from just few first poles in another one.

The numerical testing of the toy bootstrap model has shown that saturating of a given sum rule with finite number of lowest resonances may result in imbalance due to several 


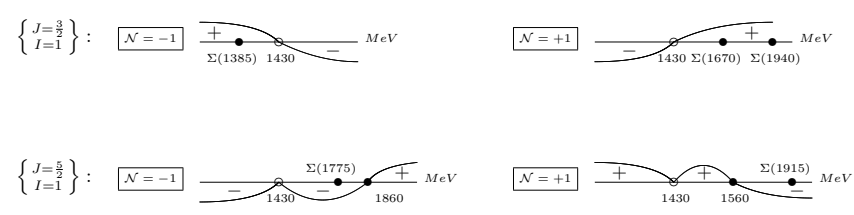

Figure 6. Signs of the contributions from $\Sigma$ hyperons with spin $j=\frac{3}{2}, \frac{5}{2}$ and normality $\mathcal{N}= \pm 1$ to the sum rule following from bootstrap constraint (5.4) with $p=k=1$.

typical reasons:

1. The sum rule belongs to the class of "bad" ones because it converges very slowly. To achieve reasonable accuracy in the process of numerical saturating, one has to take account of contributions from the very large number of distant poles;

2. It may turn out that one employs incorrect information on the asymptotic behavior of the amplitude, so that the sum rule under consideration is, in fact, divergent. This scenario was illustrated in Section 5.2;

3. Finally, the information on the resonance spectrum may be incomplete and certain light resonances, which might provide a considerable contribution, are missed. If it is possible to point out the resonance which helps to restore the balance in a given sum rule and, at the same time, does not lead to problems with saturation of another ones, this can be considered as indirect evidence in favor of the existence of such resonance.

In what follows we are going to check whether some of these scenarios can be applied to the sum rules (5.4).

The first scenario looks promising. One can easily specify the quantum numbers of heavy baryon resonances whose contributions to the sum rule (5.4) with $k=p=1$ enter with suitable signs. On Figure 6 we show the behavior of signs of contributions to the sum rule (5.4) with $p=1, k=1$ from different $\stackrel{1}{S}=-1 \Sigma$ hyperon families. We conclude that the contributions of the "tail" of heavy resonances with $J=\frac{3}{2}, \mathcal{N}=-1$ and $J=\frac{5}{2}, \mathcal{N}=+1$ may gradually compensate the large contribution from $\Sigma(1385)$. The same mechanism then also works for other sum rules from this group with $k>1$.

The Regge theory intercepts are known since 1960's and are much reliable. Thus, at first glance, the second of the above-mentioned scenarios seems to be of little use. Nevertheless, let us stress that the additional information on the high energy asymptotic behavior of invariant amplitudes of binary scattering processes at various fixed values of other kinematical variables is highly demanded. The thing is that the sum rules following from the bootstrap constraints turn out to be slowly converging in the vicinities of domains where the asymptotic regime changes. These sum rules require application of methods of convergency acceleration.

Now we are going to discuss whether we may profit from the possibility that certain resonances which could give a significant contribution to our sum rules have been for some reasons omitted. 
The sum rule (5.4) with $p=1, k=1$ may be considered as a purely baryonic one: in the meson sector only resonances with spin $j \geq 3$ may contribute. Thus to saturate it is natural to look for possible amendments to $K N$ spectrum in the baryon sector. Indeed the "world average" PDG $K N$ resonance spectrum [14] may be incomplete or imprecise both in the $u^{-}, t$ - and $s$-channel sectors of $K N$ reaction. We would like to recall (see [7]) that the numerical test of $\pi N$ bootstrap sum rules employing the more precise $\pi N$ spectrum obtained with the help of advanced coupled-channel partial wave analysis [16] rather than PDG data [14] shows considerable improvement of saturation results. We strongly suggest the application of methods employed in [16] for the $K N$ elastic scattering. A refined spectrum of $\Lambda$ and $\Sigma$ hyperons would allow the high precision tests of $K N$ bootstrap sum rules. It would be also extremely interesting to try to take account of the bootstrap constrains for resonance parameters at the level of partial wave analysis. On the other hand the problem of theoretical development of fitting procedures directly based on the effective scattering theory approach (in which the notion of resonance is rigorously defined) also awaits its solution.

Another possibility to amend the $K N$ spectrum is to assume the existence of resonances (so called $Z$ baryons) in the $s$-channel. Up to present time such a possibility has not been excluded by experiment. One of the most tempting candidates is the exotic baryon resonance with strangeness $S=+1-$ so called $\theta(1530)$. Since the prediction of its mass and width in [17] and the first experimental publications $[19,20]$ there was much interest to light and narrow exotic resonances. So far experiment does not show a clear-cut evidence of their existence. For the discussion and review of experimental situation see [21-24]. It is very interesting to see whether our sum rules can be of any use for clarifying this issue.

For example, one can try to interpret the deficit in (5.4) with $p=1, k=1$ (as well as in some other sum rules) as indirect evidence of the existence of exotic baryon (or few such baryons). To analyze this possibility in more detail we need to discuss first the characteristics (quantum numbers and widths) of the exotic resonances which could manifest themselves in our sum rules.

Unfortunately the ordinary spin- $\frac{1}{2}$ narrow exotic resonance cannot make significant contribution to our sum rules. Several recent results of data analysis estimate the possible $\theta$ decay width as $\Gamma_{\theta \rightarrow K N} \sim 1 \mathrm{MeV}$. This is consistent with the advanced theoretical estimates of $\theta$ width in the framework of Chiral Quark Soliton model [18, 25]. This makes the coupling of $\theta$ resonance to $K N$ extremely small. Its possible contribution is invisible against that of background from poorly established $\Lambda$ and $\Sigma$ hyperon resonances. This situation is quite similar to that with the contribution of recently established (see [15]) narrow resonances with $S=-1$.

Thus we conclude that the possibility to get information on spin- $\frac{1}{2}$ narrow exotic resonance from our sum rules looks unrealistic. However, the possibility that exotic resonance can be a higher spin state still is not excluded [26-29].

Among the possible choices of quantum numbers of exotic resonance $\theta J^{\mathbf{P}}=\frac{3}{2}^{-}$has the advantage that the small width of resonance is quite compatible with its significant contribution to our sum rules. On Figure 7 we show the parametric dependence of the exotic $\theta J^{\mathbf{P}}=\frac{3}{2}^{-}$decay width on the resonance mass for the fixed value of dimensionless 


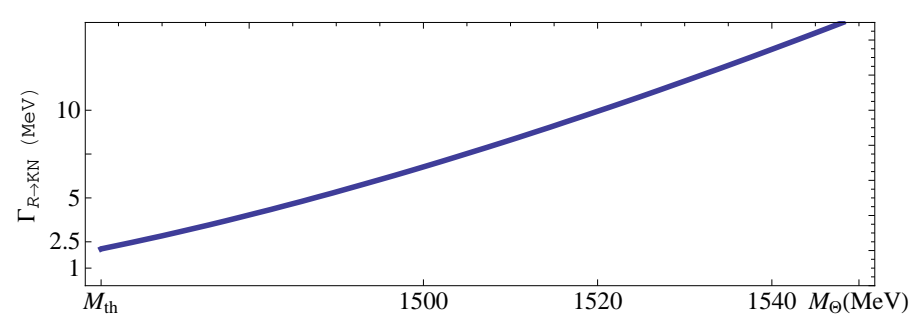

Figure 7. The parametric dependence of the decay width of $J^{P}=\frac{3}{2}^{-}$exotic resonance on its mass for the fixed value of $G_{K N \theta}=25$.

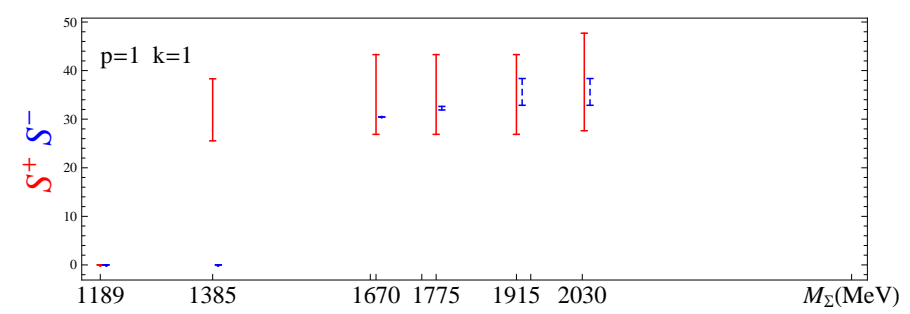

Figure 8. Numerical test of sum rule following from the bootstrap constraint (5.4) with $k=p=1$ taking into account the contribution of the exotic $S=+1$ resonance in the $s$-channel with $J^{\mathbf{P}}=\frac{3}{2}^{-}$ with $M=1530 \mathrm{MeV}$ and $\Gamma_{K N} \sim 10 \mathrm{MeV}$ Compare to Fig. 5 .

coupling constant $G_{K N \theta}=25$ (see (D.2)). Below we show that this value of $G_{K N \theta}$ allows one to saturate the sum rule (5.4) with $p=1, k=1$ by the contribution of exotics.

We conclude that for a $J^{\mathbf{P}}=\frac{3}{2}^{-}$resonance with the mass parameter $M \sim 1530 \mathrm{MeV}$ the decay width which corresponds to $G_{K N R}=25$ is about $10 \mathrm{MeV}$. It can be reduced to several $\mathrm{MeV}$ by shifting the mass parameter towards $K N$ threshold value. By the way, one can check that for the resonance with spin $J^{\mathbf{P}}=\frac{3}{2}^{+}$and mass $\sim 1530 \mathrm{MeV}$ the decay width that corresponds to $G_{K N R}=25$ is $\sim 500 \mathrm{MeV}$. That is why this choice of quantum numbers seems less favorable in our approach if we suppose the exotic resonances to be narrow and, at the same time, providing sizeable contributions to sum rules.

On Fig. 8 we show that the contribution of the exotic baryon state with $J^{\mathbf{P}}=\frac{3}{2}^{-}$ (normality $\mathcal{N}=+1$ ) above the $K N$ threshold could significantly compensate the deficit in the bootstrap constraint (5.4) with $k=p=1$. It can be shown that such a resonance could also improve the result of saturation of several other sum rules without breaking the balance in those relations which have been earlier saturated without attraction of $S=+1$ resonances.

In fact this exercise only gives an idea how the bootstrap constrains can be used for the needs of hadron spectroscopy. Obviously, for the moment we still lack the precise information on $u$ - and $t$ - channel $K N$ resonance spectrum in order to undertake the search for "realistically narrow" $(\Gamma<1 \mathrm{MeV})$ exotic $K N$ resonances. 


\section{Conclusion}

We argue that the concept of the effective scattering theory may be of considerable practical use for understanding the strong processes. The requirement of existence of a rigorously defined Dyson perturbation expansion at every fixed loop order was used in [5] - [6] as a key to solve multiple specific problems that emerge when dealing with an infinite component effective theory in the $S$-matrix sector. Fundamental requirements of covariance, unitarity, causality and crossing together with assumption on realistic asymptotic behavior of invariant amplitudes result in a set of bootstrap conditions for the physical (measurable) parameters of effective Hamiltonian. The remarkable property of renormalization invariance of the system of bootstrap conditions makes it possible the direct comparison of sum rules following from this system with experimental data.

In this paper we apply our general EST scheme to the description of hadron binary scattering in the strange sector. We construct the well-defined tree level amplitudes of $K N$ scattering in three intersecting layers $B_{s}, B_{t}, B_{u}$ and derive the system of bootstrap conditions for $K N$ resonance parameters. The numerical tests of corresponding sum rules make it possible to claim the consistency of our approach with presently known phenomenology. The additional arguments in favor of this statement will be given in a special publication on mathematical aspects of numerical testing the bootstrap conditions.

We also show that the sum rules that follow from the system of bootstrap conditions can be used as a tool to study the hadron spectrum and bring indirect evidence in favor of existence of exotic resonances in the $s$-channel of elastic $K N$ scattering.

\section{Acknowledgements}

We are grateful to A. Vereshagin for useful discussions, valuable help and advises. Also we thank M. Braun, V. Franke, S. Paston, I. Strakovsky, A. Tochin, and M. Vyazovsky for multiple discussions on various aspects of effective scattering theory approach and its application to hadron spectroscopy.

\section{A Miscellaneous}

\section{A.1 Kaon and Nucleon field parametrization}

Kaon (nucleon) fields are parameterized with the help of covariant and contravariant isotopic spinors $\left|K_{i}\right\rangle,\left|\bar{K}^{i}\right\rangle\left(\left|N_{\alpha}\right\rangle,\left|\bar{N}^{\alpha}\right\rangle\right)$ which transform under isotopic transformations as follows:

$$
I_{a}\left|K_{i}\right\rangle=\left(\sigma_{a} / 2\right)_{\cdot i}^{j \cdot}\left|K_{j}\right\rangle ; \quad I_{a}\left|\bar{K}^{i}\right\rangle=-\left(\sigma_{a} / 2\right)_{\cdot j}^{i \cdot}\left|\bar{K}^{j}\right\rangle .
$$

Here $i, j, \alpha=\{1,2\}$ stand for the isotopic spinor indices and $\sigma_{a}$ - for the Pauli matrices: $\operatorname{Tr}\left(\sigma_{a} \sigma_{b}\right)=2 \delta_{a b} \quad(a, b=\{1,2,3\})$.

The list the isotopic projecting operators for three channels of $K N$ elastic scattering reaction looks as follows: 
- s-channel:

$$
P^{s(0,1)}{ }_{\alpha i \cdot \beta}^{* \beta j}=\frac{\delta_{\alpha \cdot}^{\beta} \cdot \delta_{i}^{* j} \mp \delta_{\alpha}^{\cdot j} \cdot \delta_{i}^{*}{ }^{\beta}}{2}
$$

- $t$-channel:

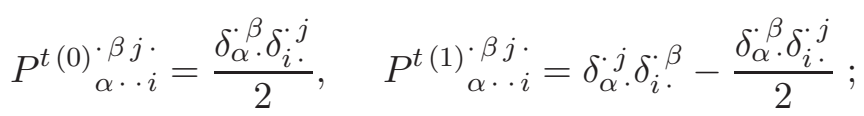

- $u$-channel:

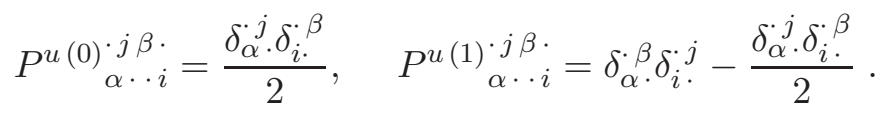

Let us also explain the isospin parametrization of resonance fields. For instance, if the resonance has isospin $I=1$ the corresponding field operator reads

$$
P_{\alpha \cdot i}^{u(1) \cdot j \beta \cdot} R_{\beta}^{\cdot i} \equiv R_{\alpha \cdot}^{(1)^{\cdot j}}=\left(\sigma_{a}\right)_{\alpha \cdot j}^{\cdot j} R^{a}
$$

Thus, for isospin 1 one may use either one isovector index $a=1,2,3$ or spinor notations with two spinor indices. The superscript "(1)" then implies the contraction with relevant isotopic projector.

\section{A.2 Asymptotic conditions}

Below we present a summary of Regge theory prescriptions for the asymptotic behavior of the invariant amplitudes $A^{ \pm}, \quad B^{ \pm}$that appear in (2.3). This behavior is determined by the known intercepts $a_{I}(0)$ of the leading Regge trajectories (see, e.g., [13]) with the cross-channel isospin $I$ :

$$
\begin{aligned}
& \left.a_{0}(s)\right|_{s=0}<0,\left.\quad a_{1}(s)\right|_{s=0}<0 \\
& \left.a_{0}(t)\right|_{t=0}=1, \quad 0<\left.a_{1}(t)\right|_{t=0}<1 ; \\
& \left.a_{0}(u)\right|_{u=0}=-0.7,\left.\quad a_{1}(u)\right|_{u=0}=-0.3 .
\end{aligned}
$$

In the boxes below we show the degrees of bounding polynomials needed to construct the corresponding Cauchy forms in various layers.

- $B_{t}:\left\{\nu_{t} \in \mathbb{C}, t \sim 0\right\}$ :

$$
\begin{aligned}
& \left.\left(2 A^{+}+A^{-}\right)\right|_{\left|\nu_{t}\right| \rightarrow \infty} \sim o\left(\left|\nu_{t}\right|^{2}\right) ; \quad N_{t}\left(2 A^{+}+A^{-}\right)=1 \\
& \left.\left(2 B^{+}+B^{-}\right)\right|_{\left|\nu_{t}\right| \rightarrow \infty} \sim o\left(\left|\nu_{t}\right|\right) ; \quad N_{t}\left(2 B^{+}+B^{-}\right)=0 \\
& \left.A^{-}\right|_{\left|\nu_{t}\right| \rightarrow \infty} \sim o\left(\left|\nu_{t}\right|\right) ; \quad N_{t}\left(A^{-}\right)=0 \\
& \left.B^{-}\right|_{\left|\nu_{t}\right| \rightarrow \infty} \sim o(1) ; \quad N_{t}\left(B^{-}\right)=-1
\end{aligned}
$$

- In two remaining layers $\left(B_{s}\right.$ and $\left.B_{u}\right)$ all invariant amplitudes possess the decreasing asymptotic behavior and neither correcting polynomials nor smooth terms are needed. 


\section{B Compact notations}

In this Appendix we summarize the notations used in main text to keep the results in a compact form. To describe the residues of the invariant amplitudes at poles corresponding to baryon resonance exchanges it is convenient to introduce two families of functions:

$$
F_{A}^{l}(M, \chi)=(M+m) P_{l+1}^{\prime}\left(1+\frac{\chi}{2 \phi}\right)+(M-m) \frac{(M+m)^{2}-\mu^{2}}{(M-m)^{2}-\mu^{2}} P_{l}^{\prime}\left(1+\frac{\chi}{2 \phi}\right)
$$

and

$$
F_{B}^{l}(M, \chi)=P_{l+1}^{\prime}\left(1+\frac{\chi}{2 \phi}\right)-\frac{(M+m)^{2}-\mu^{2}}{(M-m)^{2}-\mu^{2}} P_{l}^{\prime}\left(1+\frac{\chi}{2 \phi}\right) .
$$

$P_{l}$ stand here for the Legendre polynomials and $\phi$ is the universal Källen function:

$$
\phi \equiv|\vec{k}|_{\text {C.M.F. }}^{2}=\frac{1}{4 M^{2}}\left(M^{4}+m^{4}+\mu^{4}-2 M^{2} m^{2}-2 M^{2} \mu^{2}-2 m^{2} \mu^{2}\right) .
$$

The residues of the invariant amplitudes $(X=A, B)$ at poles corresponding to the exchange with $s$ - or $u$-channel baryon resonance with mass parameter $M$, spin $j=l+\frac{1}{2}$ and normality $\mathcal{N}$ are given (up to isotopical factors) by the expression:

$$
Y_{X}\left(j=l+\frac{1}{2}, \mathcal{N}, M, \chi\right)=G_{K N R} F_{X}^{l}(-\mathcal{N} M, \chi),
$$

where $G_{K N R}$ is a dimensionless constant

$$
G_{K N R}=g_{R}^{2} \frac{l !}{(2 l+1) ! !} \phi^{l} .
$$

The residues of the invariant amplitudes at poles corresponding to $t$-channel meson resonance exchanges with mass parameter $M$ and spin $l$ are given by the functions $W_{X}(M, l, \chi)$ :

$$
\begin{aligned}
& W_{A}(M, l, \chi)=G_{1} P_{l}(\chi)-\frac{m}{m^{2}-\frac{M^{2}}{4}} G_{2} P_{l-1}^{\prime}(\chi), \\
& W_{B}(M, l, \chi)=\frac{1}{F} G_{2} P_{l}^{\prime}(\chi),
\end{aligned}
$$

where

$$
F=\frac{1}{2} \sqrt{\left|\left(M^{2}-4 m^{2}\right)\left(M^{2}-4 \mu^{2}\right)\right|} ;
$$

and

$$
G_{1,2}=g_{K \bar{K} R} g_{N \bar{N} R}^{(1,2)} \frac{j !}{(2 j-1) ! !} F^{j}
$$

To shorten our notations we introduce the sign factor $\eta_{X}$ :

$$
\eta_{X}= \begin{cases}+1, & X=A \\ -1, & X=B\end{cases}
$$


and three sets of isotopic $(I=0,1)$ coefficients: $b_{I}^{ \pm}$- for baryons with strangeness $S=-1$, $c_{I}^{ \pm}$- for hypothetic exotic baryons with strangeness $S=+1$, and $d_{I}^{ \pm}$- for non-strange mesons:

$$
\begin{aligned}
& b_{0}^{+}=0, \quad b_{0}^{-}=+\frac{1}{2}, \quad b_{1}^{+}=1, \quad b_{1}^{-}=-\frac{1}{2} \\
& c_{0}^{+}=+\frac{1}{2}, \quad c_{0}^{-}=-\frac{1}{2}, \quad c_{1}^{+}=+\frac{1}{2}, \quad c_{1}^{-}=+\frac{1}{2} \\
& d_{0}^{+}=+\frac{1}{2}, \quad d_{0}^{-}=0, \quad d_{1}^{+}=-\frac{1}{2}, \quad d_{1}^{-}=1 .
\end{aligned}
$$

\section{The explicit expressions for generating functions}

Here we give the explicit expressions for the generating functions of the system of bootstrap constraints.

$$
\begin{aligned}
& \Phi_{X^{ \pm}}(u, s)=\sum_{B\{S=+1\}} c_{I}^{ \pm} Y_{X}(\ldots,-(\Sigma+u)) \frac{1}{s-M^{2}}-\sum_{B\{S=-1\}} \eta_{X} b_{I}^{ \pm} Y_{X}(\ldots,-(\Sigma+s)) \frac{1}{u-M^{2}} \\
& +\sum_{M} \frac{d_{I}^{ \pm}}{s+u+\Sigma}\left\{W_{X}\left(\ldots, \frac{2 s+\Sigma}{4 F}\right)-W_{X}\left(\ldots, \frac{-(2 u+\Sigma)}{4 F}\right)\right\} . \\
& \varphi_{A^{+}}(s, t)=-\sum_{B\{S=+1\}} Y_{A}(\ldots, t)\left(\frac{c_{I}^{+}}{s-M^{2}}+\frac{2 c_{I}^{+}}{t+2 \theta}+\frac{2\left(c_{I}^{+}+\frac{1}{2} c_{I}^{-}\right)(t+2 s-2 \sigma)}{(t+2 \theta)^{2}}\right) \\
& -\sum_{B\{S=-1\}}\left\{Y_{A}(\ldots, t)\left(\frac{-b_{I}^{+}}{s+t+\Sigma}+\frac{2 b_{I}^{+}}{t+2 \theta}-\frac{2\left(b_{I}^{+}+\frac{1}{2} b_{I}^{-}\right)(t+2 s-2 \sigma)}{(t+2 \theta)^{2}}\right)\right. \\
& \left.-Y_{A}(\ldots,-(s+\Sigma)) \frac{-b_{I}^{+}}{s+t+\Sigma}\right\}+\sum_{M} \frac{d_{I}^{+}}{t-M^{2}} W_{A}\left(\ldots, \frac{2 s+\Sigma}{4 F}\right) . \\
& \Psi_{A^{+}}(t, u)=\sum_{B\{S=-1\}} Y_{A}(\ldots, t)\left(\frac{b_{I}^{+}}{u-M^{2}}+\frac{2 b_{I}^{+}}{t+2 \theta}-\frac{2\left(b_{I}^{+}+\frac{1}{2} b_{I}^{-}\right)(-t-2 u+2 \sigma)}{(t+2 \theta)^{2}}\right) \\
& +\sum_{B\{S=+1\}}\left\{Y_{A}(\ldots, t)\left(\frac{-c_{I}^{+}}{t+u+\Sigma}+\frac{2 c_{I}^{+}}{t+2 \theta}+\frac{2\left(c_{I}^{+}+\frac{1}{2} c_{I}^{-}\right)(-t-2 u+2 \sigma)}{(t+2 \theta)^{2}}\right)\right. \\
& \left.-Y_{A}(\ldots,-(\Sigma+u)) \frac{-c_{I}^{+}}{t+u+\Sigma}\right\}-\sum_{M} \frac{d_{I}^{+}}{t-M^{2}} W_{A}\left(\ldots, \frac{-(2 u+\Sigma)}{4 F}\right) . \\
& \varphi_{B^{+}}(s, t)=\sum_{B\{S=-1\}}\left\{Y_{B}(\ldots, t)\left(\frac{-b_{I}^{+}}{s+t+\Sigma}+\frac{2\left(b_{I}^{+}+\frac{1}{2} b_{I}^{-}\right)}{t+2 \theta}\right)-Y_{B}(\ldots,-(s+\Sigma)) \frac{-b_{I}^{+}}{s+t+\Sigma}\right\} \\
& -\sum_{B\{S=+1\}} Y_{B}(\ldots, t)\left(\frac{c_{I}^{+}}{s-M^{2}}+\frac{2\left(c_{I}^{+}+\frac{1}{2} c_{I}^{-}\right)}{t+2 \theta}\right)+\sum_{M} \frac{d_{I}^{+}}{t-M^{2}} W_{B}\left(\ldots, \frac{2 s+\Sigma}{4 F}\right) \text {. }
\end{aligned}
$$




$$
\begin{aligned}
\Psi_{B^{+}}(t, u)= & -\sum_{B\{S=+1\}}\left\{Y_{B}(\ldots, t)\left(\frac{-c_{I}^{+}}{t+u+\Sigma}+\frac{2\left(c_{I}^{+}+\frac{1}{2} c_{I}^{-}\right)}{t+2 \theta}\right)-Y_{B}(\ldots,-(\Sigma+u)) \frac{-c_{I}^{+}}{t+u+\Sigma}\right\} \\
& -\sum_{B\{S=-1\}} Y_{B}(\ldots, t)\left(\frac{b_{I}^{+}}{u-M^{2}}+\frac{2\left(b_{I}^{+}+\frac{1}{2} b_{I}^{-}\right)}{t+2 \theta}\right)-\sum_{M} \frac{d_{I}^{+}}{t-M^{2}} W_{B}\left(\ldots, \frac{-(2 u+\Sigma)}{4 F}\right) .
\end{aligned}
$$

$$
\begin{aligned}
\varphi_{A^{-}}(s, t)= & \sum_{B\{S=-1\}} b_{I}^{-}\left\{Y_{A}(\ldots, t)\left(\frac{1}{s+t+\Sigma}-\frac{2}{t+2 \theta}\right)-Y_{A}(\ldots,-(s+\Sigma)) \frac{1}{s+t+\Sigma}\right\} \\
& -\sum_{B\{S=+1\}} c_{I}^{-} Y_{A}(\ldots, t)\left(\frac{1}{s-M^{2}}+\frac{2}{t+2 \theta}\right)+\sum_{M} \frac{d_{I}^{-}}{t-M^{2}} W_{A}\left(\ldots, \frac{2 s+\Sigma}{4 F}\right) .
\end{aligned}
$$

$$
\begin{aligned}
\Psi_{A^{-}}(t, u)= & \sum_{B\{S=+1\}} c_{I}^{-}\left\{Y_{A}(\ldots,-(\Sigma+u)) \frac{1}{t+u+\Sigma}-Y_{A}(\ldots, t)\left(\frac{1}{t+u+\Sigma}-\frac{2}{t+2 \theta}\right)\right\} \\
& +\sum_{B\{S=-1\}} b_{I}^{-} Y_{A}(\ldots, t)\left(\frac{1}{u-M^{2}}+\frac{2}{t+2 \theta}\right)-\sum_{M} \frac{d_{I}^{-}}{t-M^{2}} W_{A}\left(\ldots, \frac{-(2 u+\Sigma)}{4 F}\right) .
\end{aligned}
$$

$$
\begin{aligned}
\varphi_{B^{-}}(s, t)= & -\sum_{B\{S=-1\}} \frac{b_{I}^{-}}{s+t+\Sigma}\left\{Y_{B}(\ldots, t)-Y_{B}(\ldots,-(s+\Sigma))\right\} \\
& -\sum_{B\{S=+1\}} \frac{c_{I}^{-}}{s-M^{2}} Y_{B}(\ldots, t)+\sum_{M} \frac{d_{I}^{-}}{t-M^{2}} W_{B}\left(\ldots, \frac{2 s+\Sigma}{4 F}\right) . \\
\Psi_{B^{-}}(t, u)= & \sum_{B\{S=+1\}} \frac{c_{I}^{-}}{t+u+\Sigma}\left\{Y_{B}(\ldots,-(\Sigma+u))-Y_{B}(\ldots, t)\right\} \\
& -\sum_{B\{S=-1\}} \frac{b_{I}^{-}}{u-M^{2}} Y_{B}(\ldots, t)-\sum_{M} \frac{d_{I}^{-}}{t-M^{2}} W_{B}\left(\ldots, \frac{-(2 u+\Sigma)}{4 F}\right) .
\end{aligned}
$$

\section{Kaon-Nucleon Couplings to Resonances}

In this Appendix we give the formula and relations which are necessary to perform the numerical testing of sum rules.

First of all, we need to know the values of physical triple couplings $G_{\bar{K} N R}$ (B.4). They can be formally obtained from the known decay widths $\Gamma_{R \rightarrow \bar{K} N}$. To connect our $G_{\bar{K} N R}$ with $\Gamma_{R \rightarrow \bar{K} N}$ listed in [14] we make use of the standard relation giving particle decay width:

$$
\Gamma_{R \rightarrow \bar{K} N}=\left.\frac{1}{8 \pi M^{2}}|\vec{k}|\right|_{C M S} \sum^{\prime}\left|M_{\alpha}^{i \beta}\right|^{2}
$$


The symbol $\sum^{\prime}$ implies the summation over all allowed final states (isospin and polarization) and averaging over the initial states of the resonance.

In the case of decay of baryon resonance with the mass parameter $M$, strangeness $S=-1$ and $\operatorname{spin} J=l+\frac{1}{2}$ this sum reads:

$$
\sum^{\prime}\left|M_{\alpha}^{i \beta}\right|^{2}=\frac{1}{2 I_{R}+1} \sum_{\substack{\alpha, i \\ \beta, j}}\left(P^{u(I) \cdot j \beta \cdot}{ }_{\alpha \cdots i}^{\cdot j}\right)^{2} \underbrace{\frac{1}{2 l+1} \sum_{\substack{j=-l \ldots l \\ \lambda= \pm}}|\mathcal{M}|^{2}}_{K(l, \ldots)} \equiv \mathcal{F}_{I_{R}} K(l, M, \mathcal{N}) .
$$

The isotopic factor:

$$
\mathcal{F}_{I_{R}}=\frac{1}{2 I_{R}+1} \sum_{\substack{\alpha, i \\
\beta, j}}\left(P_{\alpha(I)^{u} \cdot j \beta}^{\cdot \beta \cdot i}\right)^{2}=\left\{\begin{array}{ll}
1, & I_{R}=0 \\
1, & I_{R}=1
\end{array} .\right.
$$

With the help of formulae of Section 2 the decay amplitude for the resonance of normality $\mathcal{N}= \pm 1$ can be written as follows:

$$
\mathcal{M}=g_{R}(i)^{l} k_{\mu_{1}} \ldots k_{\mu_{l}} \bar{u}^{+}(\lambda, p) \Gamma u^{-\mu_{1} \ldots \mu_{l}}(j, q) .
$$

The matrix $\Gamma$ is defined by (2.5). Calculation of the kinematical factor gives:

$$
K(l, M, \mathcal{N})=\left|g_{R}\right|^{2} \frac{l !}{(2 l+1) ! !} \phi^{l}\left[(M \mathcal{N}-m)^{2}-\mu^{2}\right] .
$$

Finally using (D.1), (B.4) and (B.3) we write down the formula expressing the dimensionless interaction constants (B.5) through the corresponding decay widths:

$$
G_{\bar{K} N R}=\frac{8 \pi M^{2} \Gamma_{R \rightarrow \bar{K} N}}{\phi^{\frac{1}{2}} \mathcal{F}_{I_{R}}\left[(\mathcal{N} M-m)^{2}-\mu^{2}\right]} .
$$

The summary of $\bar{K} N R$ couplings employed in our analysis for the $S=-1$ resonances obtained from the PDG data on $K N$ spectrum [14] is presented in Table 1. A formula similar to (D.2) can be written for the $K N$ coupling to $s$-channel exotic resonances.

\section{References}

[1] V. Vereshagin, Phys. Rev. D 55, 5349 (1997).

[2] A. Vereshagin and V. Vereshagin, Phys. Rev. D 59, 016002 (1999).

[3] A. Vereshagin, $\pi N$ Newsletter, 16, 426 (2002).

[4] A. Vereshagin, V. Vereshagin, and K. Semenov-Tian-Shansky, Zap. Nauchn. Sem. POMI 291, Part 17, 78 (2002) (in Russian). The English version published in J. Math. Sci., 125, Issue 2, pp. $144-158$ (2005) [arXiv: hep-th/0303242].

[5] A. Vereshagin and V. Vereshagin, Phys. Rev. D 69, 025002 (2004) [arXiv hep-th/0307256].

[6] K. Semenov-Tian-Shansky, A. Vereshagin, and V. Vereshagin, Phys. Rev. D 73, 025020 (2006). [arXiv: hep-th/0510272]. 


\begin{tabular}{|ccccl|}
\hline$R$ & $I$ & $J$ & $P(\mathcal{N})$ & $G_{\bar{K} N R}$ \\
\hline$\Lambda(1115)$ & 0 & $1 / 2$ & $+(+)$ & $206.5 \div 221.5$ \\
$\Lambda(1405)$ & 0 & $1 / 2$ & $-(-)$ & $6.4 \div 6.6$ \\
$\Lambda(1520)$ & 0 & $3 / 2$ & $-(+)$ & $16.2 \div 19.3$ \\
$\Lambda(1600)$ & 0 & $1 / 2$ & $+(+)$ & $5.7 \div 57.0$ \\
$\Lambda(1670)$ & 0 & $1 / 2$ & $-(-)$ & $0.15 \div 0.35$ \\
$\Lambda(1690)$ & 0 & $3 / 2$ & $-(+)$ & $5.2 \div 10.9$ \\
$\Lambda(1800)$ & 0 & $1 / 2$ & $-(-)$ & $1.1 \div 3.5$ \\
$\Lambda(1810)$ & 0 & $1 / 2$ & $+(+)$ & $3.1 \div 38.7$ \\
$\Lambda(1820)$ & 0 & $5 / 2$ & $+(+)$ & $10.0 \div 18.1$ \\
$\Lambda(1830)$ & 0 & $5 / 2$ & $-(-)$ & $0.04 \div 0.23$ \\
$\Lambda(1890)$ & 0 & $3 / 2$ & $+(-)$ & $0.23 \div 1.36$ \\
$\Lambda(2100)$ & 0 & $7 / 2$ & $-(+)$ & $2.7 \div 13.4$ \\
$\Lambda(2110)$ & 0 & $5 / 2$ & $+(+)$ & $1.0 \div 8.1$ \\
$\Lambda(2350)$ & 0 & $9 / 2$ & $+(+)$ & $1.0 \div 2.5$ \\
\hline \hline$R$ & $I$ & $J$ & $P(\mathcal{N})$ & $G_{\bar{K} N R}$ \\
\hline$\Sigma(1189)$ & 1 & $1 / 2$ & $+(+)$ & $141.7 \div 144.3$ \\
$\Sigma(1385)$ & 1 & $3 / 2$ & $+(-)$ & $0.8 \pm 1.2$ \\
$\Sigma(1660)$ & 1 & $1 / 2$ & $+(+)$ & $2.5 \div 35.1$ \\
$\Sigma(1670)$ & 1 & $3 / 2$ & $-(+)$ & $1.6 \div 6.0$ \\
$\Sigma(1750)$ & 1 & $1 / 2$ & $-(-)$ & $0.1 \div 1.4$ \\
$\Sigma(1775)$ & 1 & $5 / 2$ & $-(-)$ & $0.85 \div 1.27$ \\
$\Sigma(1915)$ & 1 & $5 / 2$ & $+(+)$ & $0.84 \div 5.0$ \\
$\Sigma(1940)$ & 1 & $7 / 2$ & $+(-)$ & $0.44 \div 0.79$ \\
$\Sigma(2030)$ & 1 & $9 / 2$ & $+(+)$ & $1.0 \div 2.55$ \\
\hline
\end{tabular}

Table 1. $\Lambda$ and $\Sigma$-baryon summary table. Data are taken form [14].

[7] K. Semenov-Tian-Shansky, A. Vereshagin and V. Vereshagin, Phys. Rev. D 77, 025028 (2008) [arXiv:0706.3672 [hep-ph]].

[8] P. Carruthers, Phys. Rev. 152, No 4, 1345, (1966).

[9] M. Nagels et al. Nucl. Phys B 109, 1-90 (1976).

[10] O. Dumbrajs, R. Koch, H. Pilkuhn, G. c. Oades, H. Behrens, J. j. De Swart and P. Kroll, Nucl. Phys. B 216, 277 (1983).

[11] T. Ericson, W. Weise, Pions and Nuclei (Clarendon Press, Oxford (1988)).

[12] V. de Alfaro, S. Fubini, G. Furlan, C. Rossetti, Currents in Hadron Physics (North-Holland, Amsterdam, 1973).

[13] P. D. B. Collins, An Introduction to Regge Theory and High-Energy Physics (Cambridge University Press, Cambridge, England, 1977).

[14] J. Beringer et al. (Particle Data Group), Phys. Rev. D 86, 010001 (2012)

[15] J. A. Oller, Eur. Phys. Journal A 28, 63 (2006) 
[16] R. A. Arndt, W. J. Briscoe, I. I. Strakovsky and R. L. Workman, Phys. Rev. C 74, 045205 (2006) [arXiv:nucl-th/0605082].

[17] D. Diakonov, V. Petrov, M. Polyakov, Z. Phys. A 359, 305 (1997) [arXiv: hep-ph/9703373].

[18] D. Diakonov, V. Petrov, M. Polyakov [arXiv: hep-ph/0404212].

[19] T. Nakano et al. Phys.Rev.Lett. 91, 012002 (2003) [arXiv: hep-ex/0301020].

[20] V. V. Barmin et al. [DIANA Collaboration], Phys. Atom. Nucl. 66, 1715 (2003) [Yad. Fiz. 66, 1763 (2003)] [arXiv:hep-ex/0304040];

[21] T. Nakano, Nucl. Phys. A 755, 3 (2005).

[22] K. H. Hicks, Prog.Part.Nucl.Phys. 55, 647-676 (2005) [arXiv: hep-ex/0504027].

[23] V. D. Burkert, Int.J.Mod.Phys. A21, 1764-1777 (2006) [arXiv: hep-ph/0510309].

[24] M. V. Danilov and R. V. Mizuk, Phys. Atom. Nucl. 71, 605 (2008).

[25] C. Lorce, Phys. Rev. D 74, 054019 (2006) [arXiv:hep-ph/0603231].

[26] T. Nishikawa, Y. Kanada-En'yo, O. Morimatsu and Y. Kondo, Phys. Rev. D 71, 076004 (2005) [arXiv:hep-ph/0411224].

[27] S. I. Nam, A. Hosaka and H. C. Kim, Phys. Lett. B 633, 483 (2006) [arXiv:hep-ph/0505134].

[28] S. I. Nam, A. Hosaka and H. C. Kim, Phys. Rev. C 74, 025204 (2006) [arXiv:hep-ph/0508210].

[29] P. Gubler, D. Jido, T. Kojo, T. Nishikawa and M. Oka, Phys. Rev. D 80, 114030 (2009) [arXiv:0911.2547 [hep-ph]]. 